\title{
Disk Winds of B[e] Supergiants
}

\author{
Franz-Josef Zickgraf \\ Observatoire Astronomique de Strasbourg, 11 rue de l'Université, \\ F-67000 Strasbourg, France
}

\begin{abstract}
The class of $\mathrm{B}[\mathrm{e}]$ supegiants is characterized by a two-component stellar wind consisting of a normal hot star wind in the polar zone and a slow and dense disk-like wind in the equatorial region. The properties of the disk wind are discussed using satellite UV spectra of stars seen edge-on, i.e. through the equatorial disk. These observations show that the disk winds are extremely slow, $v_{\infty} \simeq 50-90 \mathrm{~km} \mathrm{~s}^{-1}$, i.e. a factor of $\sim 10$ slower than expected from the spectral types. Optical emission lines provide a further means to study the disk wind. This is discussed for line profiles of forbidden lines formed in the disk.
\end{abstract}

\section{Introduction}

Radiation pressure is accepted as the dominant driving mechanism in the mass loss phenomenon of hot stars, especially in the upper part of the Hertzsprung-Russell (H-R) diagram. Likewise, the existence of an upper boundary of stellar luminosities in the H-R diagram is a well established observational fact (Humphreys \& Davidson 1979) which in the hot stars is believed to be related to a stability limit also caused by radiation pressure, i.e. the Eddington limit. There is now, however, increasing evidence that in addition to radiation pressure also rotation plays an important role in the mass loss process in this part of the H-R diagram.

If rotation plays a major role in the mass loss of massive stars, then effects on the circumstellar environment should be observable. It would modify the stability limit by reducing the effective gravity and thereby influence the mass loss process. The mass-loss rate should then vary with stellar latitude and therefore lead to some kind of (observable) non-sphericity.

In recent years observational evidence is indeed mounting that many hot supergiants in the upper part of the H-R diagram exhibit axial symmetry in their circumstellar environments. Likewise, indication of non-spherity has been found in their descendants, the Wolf-Rayet stars. Hence, rotation is certainly an important parameter in these stars.

Among the luminous stars the probably most spectacular object is the Luminous Blue Variable (LBV) $\eta$ Car for which recent $H S T$ images clearly showed a bipolar structure consisting of two polar lobes and an equatorial "disk". Other LBVs like R 127 and AG Car also show signs of non-sphericity (e.g. Clampin et al. 1993, Schulte-Ladbeck et al. 1994). A particularly interesting group of stars are the $B[e]$ supergiants, which most probably have 
non-spherical stellar winds caused by rotation. The B[e] supergiants represent a post-main sequence evolutionary stage of massive $\left(M \gtrsim 8 \mathrm{M}_{\odot}\right)$ stars. At this time 15 of these stars are known in the Magellanic Clouds (MCs), 4 in the SMC and 11 in the LMC (cf. Zickgraf 1998, and references therein). The observations strongly suggest that the $\mathrm{B}[\mathrm{e}]$ supergiants are characterized by a two-component stellar wind comprising in particular a disk-like, slow and dense equatorial wind which is basically distinguished from the winds observed usually in hot supergiants in the same part of the H-R diagram. An empirical model suggested by Zickgraf et al. (1985) for this group of stars is described in Sect. 2. Spectroscopic observations of the disk winds in the satellite UV are discussed in Sect. 3. Optical emission line profiles of forbidden lines originating in the disk wind are discussed in Sect. 4.

\section{Empirical model for $\mathrm{B}[\mathrm{e}]$ supergiants}

Spectroscopically and photometrically B[e] supergiants are characterized by strong Balmer emission lines, narrow permitted and forbidden low-excitation emission lines of e.g. FeII, [FeII] and [O I ], and by a strong mid-IR excess which is attributed to hot circumstellar dust with a typical temperature of $1000 \mathrm{~K}$. Most $\mathrm{B}[\mathrm{e}]$ supergiants have early-B spectral types. An important result of extensive spectroscopic observations in the optical and satellite UV region was that a subgroup of $\mathrm{B}[\mathrm{e}]$ supergiants comprising the larger fraction $(\approx 70-80 \%$ ) of these stars shows hybrid spectra (Zickgraf et al. 1985, 1986). This term means the simultaneous presence of both, narrow low-excitation lines and broad high-excitation absorption features of C IV, Si IV, and N V in the satellite UV and/or of He I in the optical region. The high ionization lines show wind expansion velocities typical for early $B$ supergiants on the order of $\sim 1000-2000 \mathrm{~km} \mathrm{~s}^{-1}$, in contrast to emission-line widths of not more than several $10 \mathrm{~km} \mathrm{~s}^{-1}$. An example of this class of objects is R 126 in the LMC which was investigated in detail by Zickgraf et al. (1985).

The smaller fraction of $\mathrm{B}[\mathrm{e}]$ supergiants does not show the signatures of a high velocity wind but only exhibits the narrow low-excitation emission lines. In most of these cases narrow and nearly unshifted absorption features of singly ionized metals similar to shell-type absorptions observed frequently in classical Be stars were found at high spectral resolution in the visual wavelength region. A typical instance of this class is R 50 in the SMC.

These properties were explained by Zickgraf et al. $(1985,1986)$ in terms of a two-component stellar wind model consisting of a radiation-driven CAKtype wind as observed in all hot high-luminosity stars (Castor et al. 1975) from the poles and an additional slow disk-forming wind from the equatorial region of the star. The model assumes that the hot and fast polar wind gives rise to the broad high-excitation absorption features whereas the lowexcitation lines and the dust are formed in a cool, dense, and slowly expanding equatorial disk wind (Zickgraf et al. 1985). 
The marked differences in the spectral appearance between individual $\mathrm{B}[\mathrm{e}]$ supergiants are interpreted in this "unified" model by assuming different aspect angles between the stars' equatorial plane and the line of sight. In this picture stars showing the broad high-excitation absorption features are viewed more or less pole-on. Those stars only showing narrow emission lines or shell-type absorption lines but no signatures of a hot high velocity wind are supposed to be seen edge-on. Support for this model comes from polarimetric observations by Magalhaes (1992). He found significant intrinsic polarisation for $\mathrm{MC} \mathrm{B}[\mathrm{e}]$ supergiants viewed edge-on according to their spectroscopic characteristics, viz. R 50 in the SMC, and the two LMC stars R 82 and Hen S22. These stars are the ideal targets to study the exotic disk winds.

\section{UV observations of the disk wind}

In order to study the properties of the outflowing disk winds we observed these three B[e] supergiants in the satellite UV (Zickgraf et al. 1996). The observations were carried out in 1991 with the International Ultraviolet Explorer (IUE) in the LWP range $(\lambda=1800-3200 \AA)$ using the high resolution mode of the spectrograph. Exposure times of up to 13 hours were required in order to obtain sufficient $\mathrm{S} / \mathrm{N}$ ratio. Sections of the spectra are displayed in Figs. 1 and 2.

Inspection of the spectra shows that they exhibit common properties, like e.g. narrow absorption features of singly ionized metals, in particular of Fe II. However, there are also individual characteristics which differ from one object to the other. Whereas in the spectrum of $\mathrm{R} 82$ the emission components are absent or only weak with the exception of $\mathrm{MgII}, \mathrm{R} 50$, and also Hen S22, exhibit much stronger emission components of the P Cygni profiles. The absorption components of $\mathrm{R} 50$ on the other hand are considerable weaker than in the two other stars, even for the Fe II of multiplet 1 . This may partly be due to a combination of a low wind velocity and the resolution of the IUE spectra. The difference between $\mathrm{R} 50$ and $\mathrm{R} 82$ is surprising because in the visual wavelength region both stars exhibit very similar spectra (Muratorio 1981, Zickgraf et al. 1986). A comparison of the spectra of R 82 and Hen S22 with those of LBVs reveals certain similarities. This is especially evident in the case of R 82. The IUE UV spectrum of this star strongly resembles the spectrum of S Dor observed during outburst phase (cf. Leitherer et al. 1985).

The wind velocities were measured from the blue edges of the absorption components. In the following all velocities are given relative to the systemic velocity taken from Zickgraf et al. (1986). The edge velocity measured for Hen S22 from the blue edges of the $P$ Cygni absorption components of strong Fe II multiplets 1,62 , and 63 is $v_{\text {edge }} \approx-120 \mathrm{~km} \mathrm{~s}^{-1}$. The centers of the P Cygni absorptions components of the strongest Fe II lines are blueshifted 

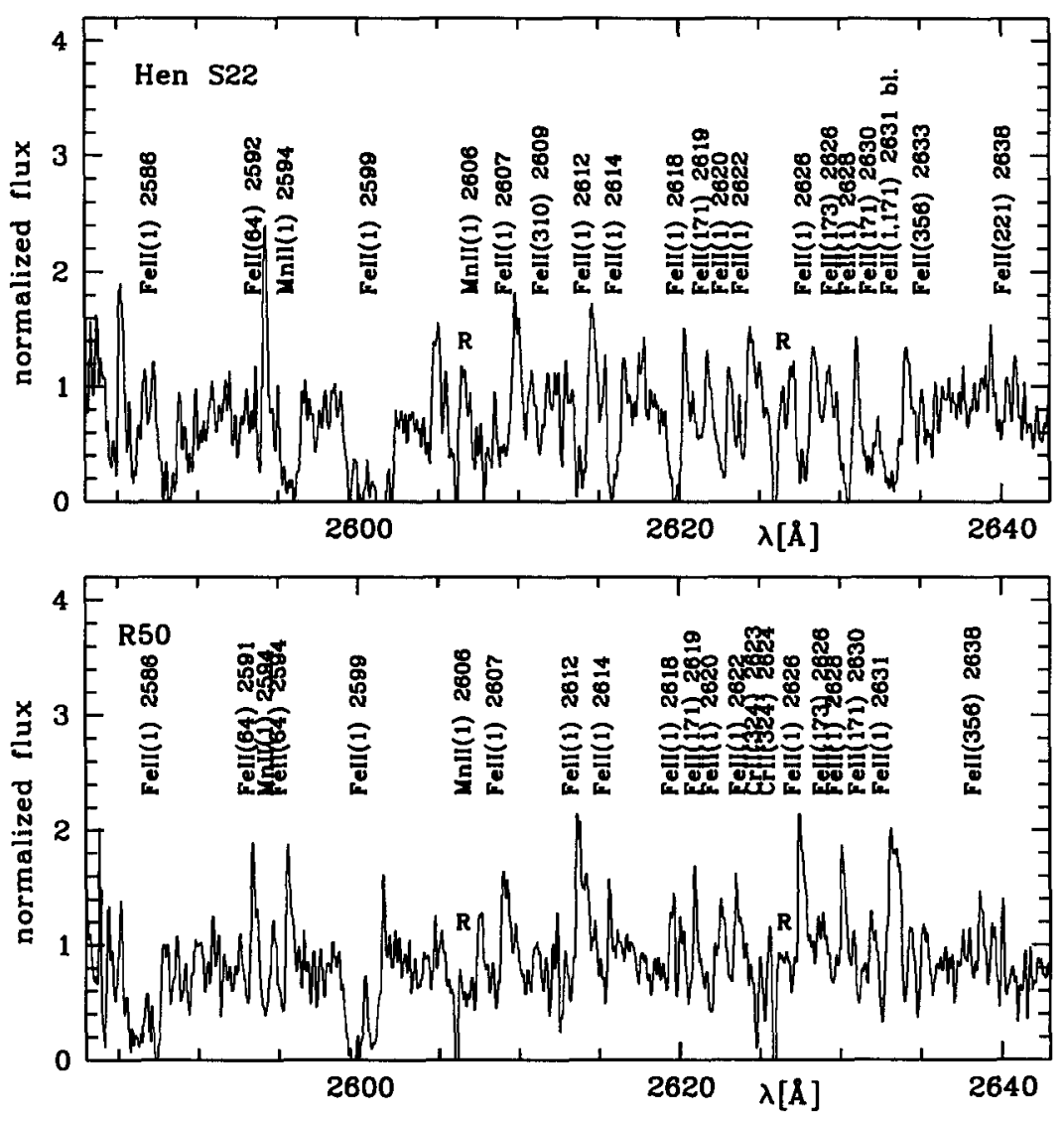

Fig. 1. Section of the IUE-LWP spectra of Hen S22 and R 50. The spectra are dominated by numerous lines of FeII. Some lines exhibit P Cygni profiles. "R" denotes reseau marks.

with respect to the systemic velocity by $v_{\exp }=-60 \mathrm{~km} \mathrm{~s}^{-1}$. The absorption lines in the spectrum of $\mathrm{R} 82$ are also shifted to the blue with respect to the systemic velocity, however, slightly less than for Hen S22. The edge velocity is $v_{\text {edge }} \approx-100 \mathrm{~km} \mathrm{~s}^{-1}$ for the strongest lines. The expansion velocity at the centers of these lines is $v_{\exp }=-40 \mathrm{~km} \mathrm{~s}^{-1}$ only. Although the absorption components of the $\mathrm{P}$ Cygni profiles of $\mathrm{Fe} I \mathrm{I}$ in the spectrum of $\mathrm{R} 50$ are weaker than in the previous two stars we could nevertheless measure the expansion and edge velocities for several Fe II lines. An edge velocity of $v_{\text {edge }}=-75$ $\mathrm{km} \mathrm{s}^{-1}$ was determined from the blue edges of the strongest lines of $\mathrm{Fe}$ II. The expansion velocity measured at the line centers of Fe II is $-27 \mathrm{~km} \mathrm{~s}^{-1}$.

The edge velocities overestimate the terminal wind velocity due to turbulent motions in the winds. Improved values for the terminal velocities of the 


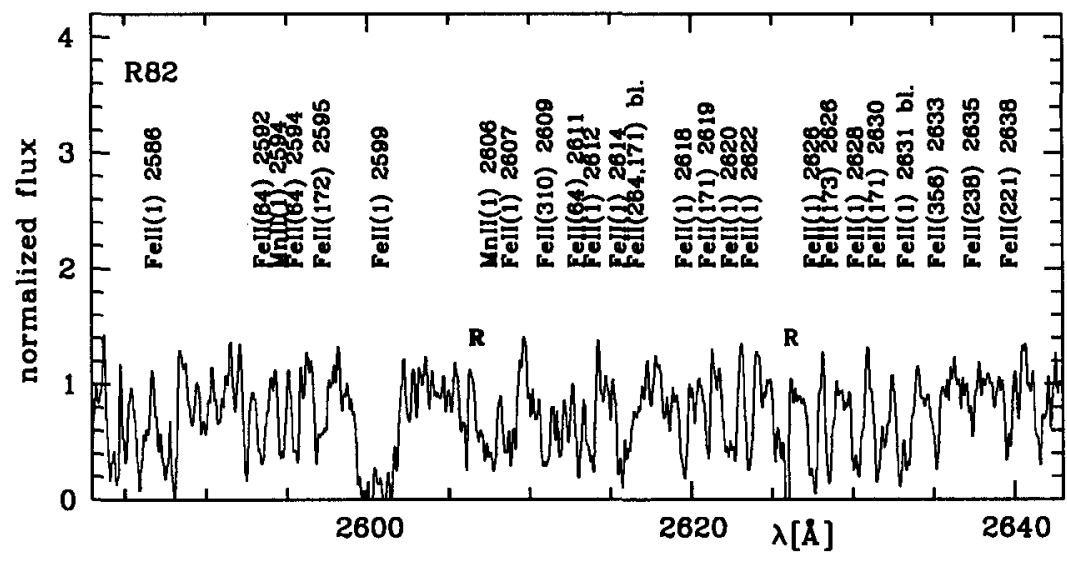

Fig. 2. Section of the IUE-LWP spectra of $R 82$. The spectrum is dominated by numerous narrow absorption lines of Fe II, very similar to $\mathrm{S}$ Dor.

winds were derived from line fits for the Fe II lines. We used the SEI method for the calculation of the line profiles (Lamers et al. 1987) which takes this effect into account. A disk geometry was assumed with a disk opening angle of $30^{\circ}$. Turbulence velocities $v_{\mathrm{D}}$ are on the order of 0.2 to $0.3 v_{\infty}$. The terminal velocity is then $v_{\infty} \simeq v_{\text {edge }}-2 v_{\mathrm{D}}$. This leads to terminal velocities smaller than measured from the blue absorption edges, i.e for Hen $\mathrm{S} 22$ $v_{\infty}=85 \mathrm{~km} \mathrm{~s}^{-1}$, for R $82 v_{\infty}=70 \mathrm{~km} \mathrm{~s}^{-1}$, and for R $50 v_{\infty}=50 \mathrm{~km} \mathrm{~s}^{-1}$.

The results are summarized in Tab. 1 . They show that the disk winds are rather extreme compared to other object classes like LBVs, and B-A superand hypergiants. The velocities derived for the $\mathrm{B}[\mathrm{e}]$ supergiants are about a factor of 10 smaller than expected from their spectral types. They have even slower winds than the LBVs and the A-type hypergiants.

Adopting a ratio $v_{\infty} / v_{\text {esc }} \simeq 1.3$ (Lamers et al. 1995) allows to estimate $\log g_{\text {eff }}$ and from this quantity with mass $M \simeq 2 / 3 M_{\mathrm{ZAMS}}, \Gamma=\Gamma_{\text {rad }}+\Gamma_{\text {rot }}$, where $g_{\text {eff }}=(1-\Gamma) g_{\mathrm{grav}} . \Gamma_{\mathrm{rad}}$ and $\Gamma_{\text {rot }}$ are due to radiation pressure and rotation, respectively. This leads to $\log g_{\text {eff }}$ value of $0.2-0.7$. Rotation at a speed of $\leqslant 220 \mathrm{~km} \mathrm{~s}^{-1}$, i.e. $70-80 \%$ of break-up velocity, would be sufficient to cause these low effective gravities.

\section{Emission lines from the disk wind}

The properties of the disk wind can also be studied using the emission line profiles of the low-excitation lines which originate in the disk. Of particular interest are forbidden transitions because they are optically thin and therefore radiation transfer does not complicate the interpretation of the line profiles. For this purpose selected lines, mainly forbidden lines of [OI ], [Fe II], [N II], 
Table 1. Wind velocities of the B[e] supergiants as derived from the blue absorption edges, $v_{\text {edge }}$, and $v_{\infty}$ derived from the SEI line fits together with velocities obtained for LBVs and A-type hypergiants. Included are also average wind velocities of normal B- to A-type supergiants.

\begin{tabular}{|c|c|c|c|c|}
\hline$\overline{\text { Star }}$ & Sp. type & $\begin{array}{l}v_{\text {edge }} \\
{\left[\mathrm{km} \mathrm{s}^{-1}\right]}\end{array}$ & $\begin{array}{l}v_{\infty} \\
{\left[\mathrm{km} \mathrm{s}^{-1}\right]}\end{array}$ & reference \\
\hline Hen S22 & $\mathrm{B}[\mathrm{e}] 0-0.5$ & 120 & 85 & Zickgraf et al. (1996) \\
\hline $\mathrm{R} 82$ & $\mathrm{~B}[\mathrm{e}] 2-3$ & 100 & 70 & $"$ \\
\hline R 50 & $\mathrm{~B}[\mathrm{e}] 2-3$ & 75 & 50 & $"$ \\
\hline $\mathrm{R} 71$ & LBV & 127 & & Wolf et al. (1981) \\
\hline S Dor & LBV & 140 & & Leitherer et al. (1985) \\
\hline R 127 & LBV & 150 & & Wolf et al. (1988) \\
\hline R 45 & $\mathrm{~A} 3 \mathrm{Ia}-\mathrm{O}$ & 200 & & Stahl et al. (1991) \\
\hline \multirow[t]{5}{*}{ R 76} & A0Ia-O & 200 & & $"$ \\
\hline & $\mathrm{B} 0$ & 1970 & & Panagia \& Macchetto (1982) \\
\hline & $\mathrm{B} 1$ & 830 & & $"$ \\
\hline & B5 & 500 & & $"$ \\
\hline & A0 & 185 & & $"$ \\
\hline
\end{tabular}

but also permitted lines of Fe II, were observed in a sample of MC B[e] supergiants with the coudé echelle spectrometer (CES) at the 1.4m CAT at ESO, La Silla. The spectra have a spectral resolution of $R=50000$, i.e. a velocity resolution of $\Delta v=6 \mathrm{~km} \mathrm{~s}^{-1}$.
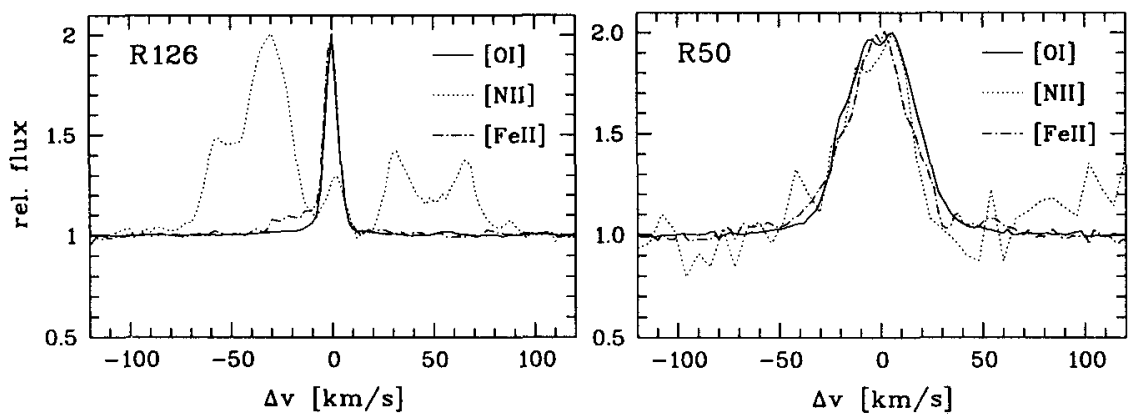

Fig. 3. Profiles of forbidden lines of R 126 (pole-on) and R 50 (edge-on), observed with a velocity resolution of $6 \mathrm{~km} \mathrm{~s}^{-1}$. The profiles have been normalized to the maximum line flux. Note the complex structure of the profile of [N II] of R 126. R 50 shows a split $[\mathrm{OI}]$ profile $\left(\Delta v=15 \mathrm{~km} \mathrm{~s}^{-1}\right)$.

The high-resolution spectra showed that the structure of the line forming zone may be rather complex, probably more complex than assumed in the empirical model discussed in Sect. 2. As examples line profiles of the pole-on 
star R 126 and the edge-on star R 50 are displayed in Fig. 3. The [N II] profile of $\mathrm{R} 126$ is rather complicated. It is split in five components, one unshifted at the systemic velocity, and two on the red and the blue side, respectively. The $[\mathrm{OI}]$ and [Fe II] lines show only one component at the systemic velocity. The profile of $[\mathrm{N}$ II] can at least qualitatively be understood if the splitting of the blue and red component is neglected. R 126 is very likely seen poleon. Assuming a radially expanding disk wind with a velocity on the order of $100 \mathrm{~km} \mathrm{~s}^{-1}$, a disk opening angle of $30^{\circ}$, and an inclination angle of $0^{\circ}$, a velocity component perpendicular to the plane of the disk of about $50 \mathrm{~km} \mathrm{~s}^{-1}$ would occur. The blue and the red emission components could then be formed in layers at the edges of the disk. Disk expansion perpendicular to the disk could also explain the observed absorption dip in $\mathrm{H} \alpha$ (Zickgraf et al. 1985). The unshifted component of [N II], and the [O I] and [Fe II] lines should originate close to the plane of the disk. Rotation could also play a role in the line forming region. $\mathrm{R} 50$ shows a split line profile of [O I]. The line splitting is $15 \mathrm{~km} \mathrm{~s}^{-1}$. It was interpretated by Zickgraf (1988) as being due to a rotating disk seen edge-on. Although the general appearance of the profiles can be qualitatively understood with the disk model the complex line profile of [N II] of R 126 indicates a more complicated structure than that of a simple disk.

\section{References}

Castor J.P., Abbott D.C., Klein R.I., 1975, ApJ 195, 157

Clampin M., Nota A., Golimowski D.A., Leitherer C., Durrance S.T., 1993, ApJ Letters 410 , L35

Humphreys R.M., Davidson K., 1979, ApJ 232, 409

Lamers H.J.G.L.M., Cerruti-Sola M. Perinotto M., 1987, ApJ 314, 726

Lamers H.J.G.L.M., Snow T.P, Lindholm D.M., 1995, ApJ 455, 269

Leitherer C., Appenzeller I., Klare G., Lamers H.J.G.L.M. Stahl O., Waters, L.B.F.M., Wolf B., 1985, A\&A 153, 168

Magalhaes A.M., 1992, ApJ 398, 286

Muratorio G. 1981, A\&AS 43, 111

Panagia N., Macchetto F., 1982, A\&A 106, 266

Schulte-Ladbeck R.E., Clayton C.G., Hillier D.J., Harries T.J., Howarth I.D., 1994, ApJ 429, 846

Stahl O., Aab O., Smolinski J., Wolf B., 1991, A\&A 252, 693

Wolf B., Appenzeller I., Stahl O., 1981, A\&AS 74, 239

Wolf B., Stahl O., Smolinski J., Cassatella A., 1988, A\&A 103, 94

Zickgraf F.-J., 1988, in: L. Bianchi and R. Gilmozzi (eds.) Mass Outflows from Stars and Galactic Nuclei, Kluwer Academic Publishers, Dordrecht, p. 211

Zickgraf, F.-J., 1998. In: B[e] stars, eds. A.M. Hubert and C. Jaschek, Kluwer Academic Publishers, in press

Zickgraf, F.-J., Wolf, B., Stahl, O., Leitherer, C., Klare, G., 1985, A\&A 143, 421

Zickgraf, F.-J., Wolf, B., Stahl, O., Leitherer, C., Appenzeller, I., 1986, A\&A 163, 119 
Zickgraf F.-J., Humphreys R.M., Lamers H.J.G.L.M., Smolinski J., Wolf B., Stahl O., 1996, A\&A, 315, 520

\section{Discussion}

M. Friedjung: Can rotation affect the line profiles? You might have a combination of rotational plus wind line broadening and the relation between the emission widths and the wind velocity could be coincidental.

F.-J. Zickgraf: Yes, rotation can affect the line profiles. I have in fact modelled emission-line profiles assuming a rotating disk in order to explain doublepeaked profiles (Zickgraf 1988, in: Mass Outflows from Stars and Galactic Nuclei, L. Bianchi and R. Gilmozzi, Kluwer, p. 211). However, for the fitting of the UV P Cygni profiles we have assumed purely radial expansion.

A. Maeder: First, I would like to emphasise that your two-component stellar wind is quite consistent with the prediction of the so-called von Zeipel theorem for rotating stars. Now, can you give estimates for the ratio of the polar to equatorial mass loss rates? This is quite important for stellar evolution, because polar mass loss does not remove angular momentum while equatorial mass loss does.

F.-J. Zickgraf: The mass flux ratio between the equatorial and polar wind is likely on the order of a factor of 10 , maybe even higher.

S. Shore: You know that there is another way to get displaced narrow lines: take $\beta \ll 1$ (say, 0.2 or so) and you will get narrow lines (e.g., slab calculations for MWC 560), so your assumption of $\beta \sim 4$ may be too restrictive. Also, why are you throwing away the absolute flux calibration of the IUE data? Normalisation can be very misleading.

F.-J. Zickgraf: The value $\beta \sim 4$ is suggested by the similarity of the $B[e]$ spectra with those of A hypergiants for which $\beta \sim 4$ was derived. The flux calibration was not thrown away. However, for the line fitting procedure we were primarily interested in the ratio of line flux to continuum flux $\left(F_{\lambda} / F_{c}\right)$ rather than calculating absolute fluxes.

A. Moffat: In your cartoon you showed that dust forms in the disk. Although no one knows (in detail) how to form dust in hot environments, all would agree that you need high compression (plus shielding). So would this not suggest that the disk is quite thin?

F.-J. Zickgraf: The assumption that the density in the disk is constant perpendicular to the plane of the disk is very likely too simple. It seems more realistic to assume a density enhancement toward the mid-plane of the disk, i.e., a decreasing density perpendicular to the disk plane. Therefore, close to the mid-plane of the disk much higher densities could be found than implied by the simplified picture presented in the cartoon. 
J. Bjorkman: Your Fe II mass-loss rate places a lower limit on the electron scattering optical depth in the disk. If $\tau_{e s} \sim 1$ and the opening angle is about $30^{\circ}$ then the polarisation will be around $6 \%$ or so. Is this consistent with polarisation observations of $\mathrm{B}[\mathrm{e}]$ supergiants?

F.-J. Zickgraf: The polarisation is as much as $2-3 \%$ (cf. also Magalhães \& Rodrigues, these proceedings).

P. Stee: How do you determine the continuum level in order to obtain UV edges? Do you take into account the underlying photospheric lines in your simulations? We have developed a "two component" radiative wind model (these proceedings) where the wind is pushed by optically thin lines at the equator and optically thick ones in the polar regions leading to a $v_{\infty}^{\text {pole }} / v_{\infty}^{\text {eq }} \sim$ 10 with $v_{\infty}^{e q} \sim 100-200 \mathrm{~km} / \mathrm{s}$ and a mass flux ratio of $\Phi_{e q} / \Phi_{\text {pole }} \sim 30$, in agreement with your observations (cf. Stee et al. 1995, A\&A, 300, 219; Stee 1998, A\&A, in press).

F.-J. Zickgraf: The normalisation was done in the two-dimensional orderwavelength space of the high-resolution IUE echelle spectra. Photospheric lines were not taken into account for the profile calculations. Photospheric $\mathrm{Fe}$ II is not expected to contribute significantly in early B supergiants.

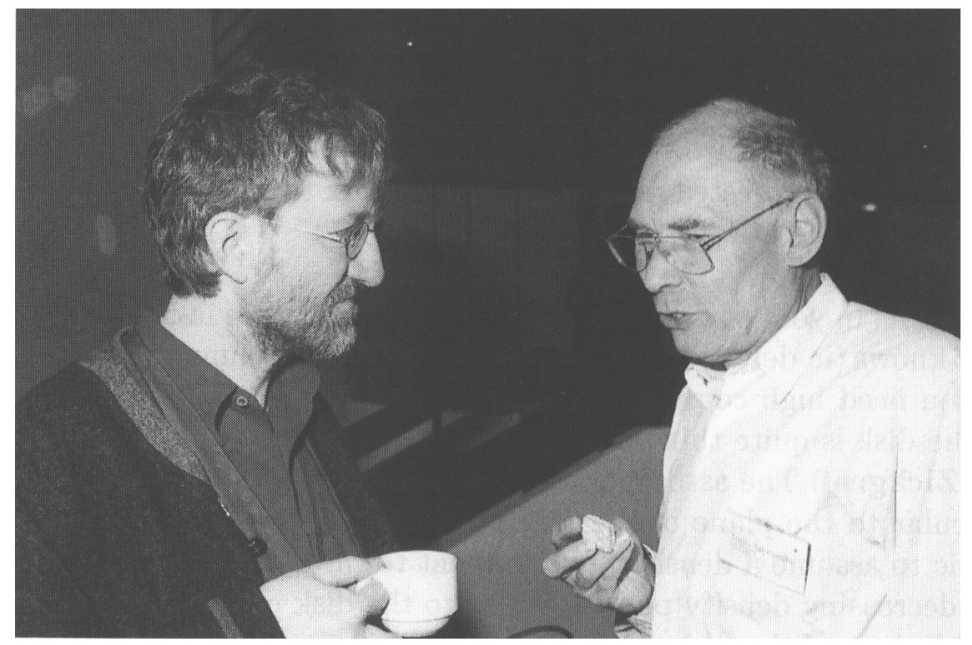

Franz-Josef Zickgraf and Immo Appenzeller 